\title{
Process Monitoring with Disposable Chemical Sensors Fit in the Framework of Process Analysis Technology (PAT) for Innovative Pharmaceutical Development and Quality Assurance
}

\author{
Stefan Spichiger and Ursula E. Spichiger-Keller*
}

\begin{abstract}
The innovative principle of enzymatic sensors applied to monitor the feeding process in disposable bioreactors is described. Innovative is the type of enzyme immobilized within the 'paste' to monitor L-glutamate. Innovative is the application of the miniaturized disposable sensor developed at C-CIT AG for continuous monitoring. The sensor allows the amount of the digested nutrient to be estimated from the amperometric signal. Innovative is the wireless signal transduction between the sensor mounted to the bioreactor and the signal receiver. An example of a process control run is given and, also, the biocompatibility and the specifications of the biosensors. The comparison of results evaluated by different analytical methods is discussed.
\end{abstract}

Keywords: Biosensors $\cdot$ Continuous monitoring $\cdot$ Electrochemistry $\cdot \beta$-D-Glucose $\cdot L-G l u t a m a t e$

\section{Introduction}

According to the FDA Guidance for Industry (PAT Guidelines $\left.{ }^{[1]}\right)$, Process Analytical Technology (PAT) "is intended to support innovation and efficiency in pharmaceutical development". The Agency encourages manufacturers to use the PAT framework to develop and implement effective, innovative approaches in development, manufacturing and quality assurance.

The sensors described in this article represent one possible solution to a critical requirement in biopharmaceutical systems, namely the ability to analyze and control critical cultivation parameters with realtime measurements during the cultivation process. Such a critical cultivation parameter is the supply of carbohydrate such as glucose as a nutrient and source of carbon. Monitoring the concentration of glucose in a cell culture medium allows cell growth to be surveyed directly in the medium and

${ }^{\star}$ Correspondence: Prof. Dr. U. E. Spichiger-Keller C-CIT AG

Einsiedlerstr. 29

$\mathrm{CH}-8820$ Wadenswil

Tel.: +41434778555

Fax: +41434778557

E-mail: ursula.spichiger@c-cit.ch constitutes the one parameter which correlates inversely with the cell density.

To fulfill these requirements, novel analytical systems and devices have been developed and are now commercially available. Such devices include single-use sensors, which are either integrated in the disposable bioreactor or included in the cover. They are then discarded together with the bioreactor. They provide a continuous signal and allow information about the status of a cell culture to be gathered at any time. In contrast to batch analysis, such as HPLC, electrochemistry and wet chemical analysis, ${ }^{[2-4]}$ this type of bioprocess control reduces the risk of contamination and the effect of shear stress on the cells.

An example of such a biosensor, CITSens Bio, is illustrated in Fig. 1a. The CITSens Bio monitors the consumption of $\beta$-D-glucose and L-glutamate as feed parameters and/or the production of L-lactate as a growth inhibiting metabolite during the bioprocess.

The goal of developing this sensor was to measure glucose consumption as well as other parameters continuously, to provide disposable tools at a reasonable price and to make the production process more effective. The production process can be terminated in the phase of maximum rate of product yield. Continuous monitoring also reduces the risk of contamination. No staff time is needed to sample aliquots of the medium in the flow-bench for external analysis. Moreover the disposable bioreactor does not have to be removed from the incubator and exposed to ambient conditions, which disturbs the production process.

\section{Experimental}

\subsection{Quality Assessment of Sensors: Chemicals and Reagents}

Chemicals for calibration solutions: D(+)-glucose $>99.5 \%$ (HPLC), L-glutamic acid $>99.0 \%$, L-(+)-lactic acid 98\%, lithium chloride anhydrous, puriss. p.a. (low in sodium) all from Sigma-Aldrich, Switzerland. Imidazol buffer solution, doubly distilled water.

Chemicals for $\mathrm{pH}$-buffer solutions: Imidazol puriss. $\geq 99.5 \%$, solutions $100 \mathrm{mmoll}^{-1}$ $\left(25^{\circ} \mathrm{C}\right.$ ) adjusted to $\mathrm{pH} 7.0$ by hydrochloric acid 37\% (puriss.p.a., Sigma Aldrich Chemie GmbH, CH-9471 Buchs, Switzerland). To obtain the conductivity required, lithium chloride was added (4.239 $\mathrm{g} \mathrm{l}^{-1}$, $100 \mathrm{mmol} \mathrm{l}^{-1}$ ).

Chemistry of the 'paste'. The chemistry, which includes the enzyme and the additives, is confidential. The recipe to prepare the paste is owned by C-CIT AG.

Calibration procedure: zero point is referred to buffer solution and is very reproducible (instrumental default); D-(+)-glucose, calibration range: 1 to $30 \mathrm{mmol} \mathrm{l}^{-1}$; L-(-)-glutamic acid, range: 1 to $30 \mathrm{mmoll}^{-1}$; L-(-)-lactic acid, range: 1 to $30 \mathrm{mmol} \mathrm{l}^{-1}$ CHO cells: Chinese Hamster Ovarian Cells

Cell culture medium: buffered aqueous solution consisting of all nutrients (amino 


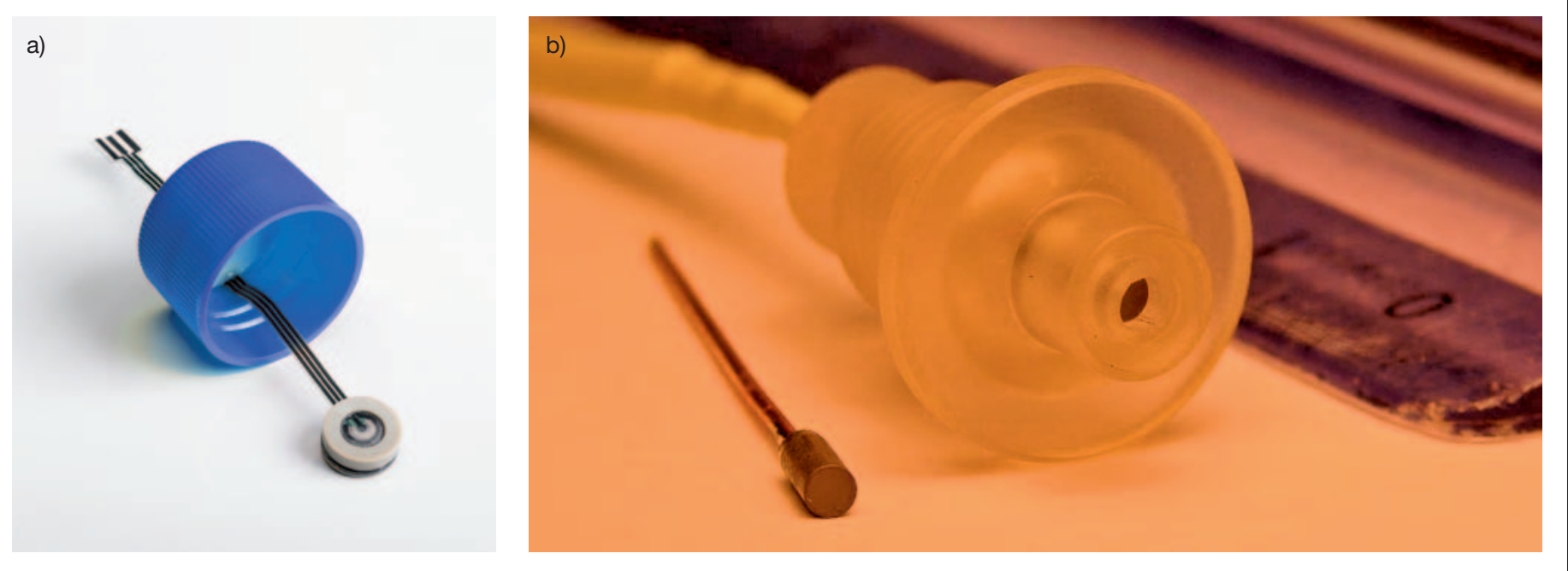

Fig. 1. a) CITSens Bio Sensor integrated in the cover of a disposable bioreactor. b) Head of the working electrode which is used in the flowthrough system for chronoamperometric experiments. ${ }^{[5]}$ The center of the electrode is coloured black and contains the biosensor 'paste' with the immobilized enzyme.

acids, carbohydrates and fatty acids) to feed the Chinese Hamster Ovarian Cells, to make them grow and to duplicate them in an exponential way (confidential composition). The medium contains an indicator to indicate the $\mathrm{pH}$ when it switches to acid conditions. The special medium used for the experiments shown in Fig. 3 (Section 3.3) was FMX-8 by Dr. Feruccio Messi, ${ }^{[6]}$ cell culture technologies, CH-6929 Gravesano, Switzerland (www.cellculture.com).

\subsection{The Enzymes: GOD, LOD and L-Glutamate Oxidase}

$\beta$-D-Glucose oxidase from Aspergillus niger, 190-210 units $\mathrm{mg}^{-1}$ (GOD, EC 1.1.3.4), L-glutamate oxidase from streptomyces sp. (LGOX, EC 1.4.3.11), L-lactate oxidase from Pediococcus sp., $\geq 20$ units $\mathrm{mg}^{-1}$ (LOD, EC 1.1.1.27). LOD and GOD are from Sigma Aldrich Chemie $\mathrm{GmbH}$, CH-9471 Buchs, Switzerland. LGOX was received from Hitoshi Kusakabe. ${ }^{[7,8]}$ L-Glutamate oxidase catalyses the deamination of L-glutamate. The mature enzyme used in the sensor of C-CIT AG, is derived from a precursor of LGOX. In nature, LGOX is expressed as a precursor in an incompletely active form. When digested by an endopeptidase, the mature enzyme with a total molecular mass of approx. $70 \mathrm{kD}$ and the hexadimeric structure $\alpha_{2} \beta_{2} \gamma_{2}$ is yielded.

LGOX has high substrate specificity and high stability (thermal stability $\sim 80^{\circ} \mathrm{C}$, kcat $\left.75 \mathrm{~s}^{-1}, \mathrm{Km}=0.23 \mathrm{mM}\right)$. The crystal structure of the LGOX has been published and the amino acid sequence elucidated. ${ }^{[8]}$

\subsection{The Biosensor CITSens Bio}

The principal feature of the CITSens Bio sensor is a miniaturized, screen-printed electrode (Fig. 1a), comprising a system with three electrodes for amperometric detection of the glucose-turnover and the current transmitted to the anode (working, counter and reference electrode). The screen-printed electrode is manufactured by ALGRA AG, CH-5634 Merenschwand. This three-electrode system ensures a reliable electrical signal with long-term stability. The chemical components incorporated into a so-called 'paste' are deposited onto the active field of the working electrode. The 'paste' includes the enzyme and a scarcely soluble organic conductor. The enzyme is cross-linked to a bulk protein and is hence immobilized in this network. The immobilization process itself has an antimicrobial effect. A dialysis membrane with a low molecular cutoff is cast over the sensing head to create a barrier between the sensor and the cultivation medium. In addition, the dialysis membrane limits the uptake of the enzyme's substrate by the sensor and defines the concentration gradient of the substrate between the bulk medium and the active field of the sensor surface. The sensor device is mounted on the screwed-on cover of the bioreactor (Fig. 2) and packaged in a thermoplastic bag for gamma-sterilization and shipping. At the point of use, the thermoplastic bag is opened and the sensor is aseptically connected to the bioreactor (flow-bench).

\subsection{Continuous Monitoring, Wire- less Transmission of the Signal and Signal Sampling Frequency}

The amperometric signal provided by the biosensor (see Fig. 2) is detected by the radio-frequency beamer (Bio Beamer) and wirelessly transmitted to the receiver $\left(\mathrm{ZOMOFI}^{\circledR[9]}\right)$. Both devices are from Albis Technologies AG, CH-8047 Zürich. The Bio Beamer contains the electric measuring cycle and the power supply and was specifically developed by Albis Technology according to the specifications of

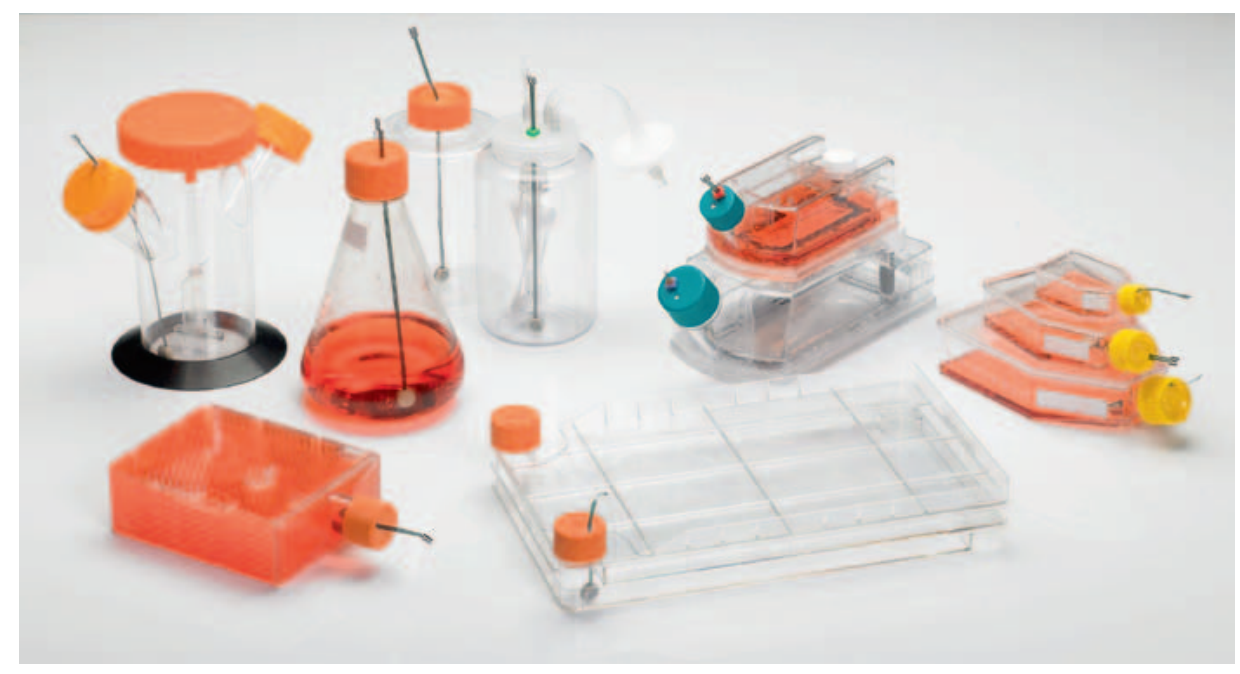

Fig. 2. CITSens Bio mounted on various disposable bioreactors (from left to right: HyperFlask, spinner bottle, shake flask, roller bottle, SuperSpinner D1000, CeLLines, CellFactory, t-flasks). 
C-CIT AG. Each sensor/Bio Beamer is identified by a specific tag, which is recognized by the ZOMOFI. The receiver, which is able to $\log$ the responses of up to 1000 sensors, is connected to a PC to facilitate data processing and storage. Each device is identified on the PC by its specific tag and the performance of each sensor is monitored continuously. In order to reduce the number of collected data points, data are acquired based on the desired sampling rate (every $20 \mathrm{~s}$ or longer). The results from several bio-monitoring experiments using disposable reactors are discussed in the following section.

\section{The Basics of the Biosensor CIT Sens Bio}

\subsection{A Biosensor with Direct Elec- tron Transfer from $\beta-D-G l u c o s e$, L-Glutamate and L-Lactate to the Electrode}

The CITSens Bio sensors utilize an enzymatic oxidation process and electron transfer from the specific substrate to the electrode (anode) via a chemical wiring process. Oxidation is catalyzed by an enzyme specific for $\beta$-D-glucose, L-lactate or L-glutamate, an organic conductor ${ }^{[2]}$ and an exceptionally low applied voltage $(<100 \mathrm{mV})$. The sensor is therefore not affected by the oxygen concentration and produces an exceptionally low concentration of side-products, such as peroxide and ammonia. Due to the low applied voltage, the sensor has a high selectivity to interfering components and direct oxidation of them. The working principle of this sensor is different from that of a number of well-known alternatives currently on the market, which depend on having a sufficient supply of oxygen for their operation as they measure the hydrogen peroxide $\left(\mathrm{H}_{2} \mathrm{O}_{2}\right)$ produced during the bioprocess.

\subsection{Substrate Consumption by the Biosensor for $\beta-D-$-Glucose as an Example}

The version of the sensor design used for the experimental evaluation of glucose consumption by the biosensor differs slightly from the commercialized tool (Fig. 1b). The chronoamperometric experiments are described in detail by Wunderli and Andres. ${ }^{[10]} \mathrm{A}$ voltage of $100 \mathrm{mV}$ is applied between the biosensor (working electrode) and the counterelectrode ${ }^{[5,11]}$ of a rotating disk electrode, and similarly of an electrode mounted on a flow-through system. ${ }^{[5,10]}$ The voltage is stabilized with an $\mathrm{Ag} /$ $\mathrm{AgCl}$-reference electrode. In chronoamperometry, the current $\left(10^{-6}\right.$ to $\left.10^{-5} \mathrm{~A}\right)$ is monitored over time (s) and correlates with the substrate turnover of the enzyme at the surface. A standard D-(+)-glucose

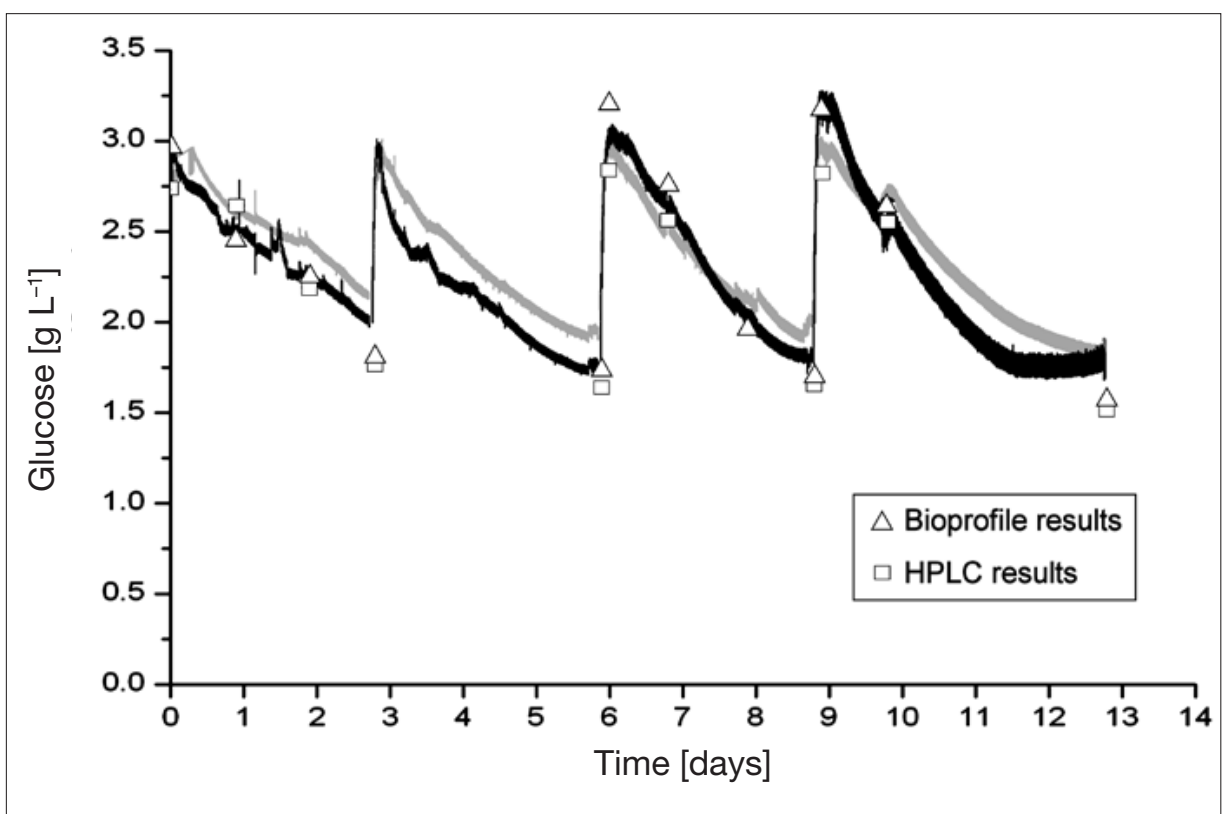

Fig. 3. Glucose monitoring in a $\mathrm{CHO} \mathrm{K} 1$ cell suspension culture over a two-week period. The graph illustrates the results of four growth cycles including three passages of cells. The two biosensors exhibit an adequate correlation. The results of HPLC (squares) and BioProfile ${ }^{[4]}$ (triangles) are indicated.

concentration of $5 \mathrm{mM}$ was used in these experiments. Based on Faraday's Law, it is feasible to estimate the consumption of substrate relative to the geometry of the electrode exposed to the cell culture medium and the specific activity of the enzyme. The medium is thermostatted to 37 ${ }^{\circ} \mathrm{C}$ and buffered to $\mathrm{pH} 7.0$ with imidazole buffer. The conductivity is stabilized with $\mathrm{LiCl}$ solution $(0.1 \mathrm{M})$. In all experiments run with the rotating disk electrode and the continuous flow system, it was evident that the current was robust to the flux at the electrode surface. Therefore, the result yielded from these experiments can be transposed to the situation in a shaken or stirred bioreactor. It should be noted that the applied voltage, the glucose specificity and diffusion gradient of the enzyme substrate between bulk medium and sensor field depend on each other and influence the quality of the analytical result and the detection range.

For an electrode with a surface area of $1 \mathrm{~mm}$ in diameter (see Fig. 1b) and $5 \mathrm{mM}$ glucose solution (D-(+)-glucose), 1.1.10 $( \pm 0.03) \mathrm{A}$ per $\mathrm{mm}^{2}$ area over 100 seconds were measured. For the commercial biosensor with a surface area of $0.5 \mathrm{~mm}^{2}$ area, the glucose consumption in reality is estimated to be in the range of $<10^{-12} \mathrm{~mol} \beta$-Dglucose per $\mathrm{s}$ and in the range of $<10^{-5}$ to $10^{-6}$ mol glucose over $7-14$ days. In comparison, the bulk glucose concentration of a cell culture medium is in the range of $1-5.10^{-2}$ mol per $1000 \mathrm{ml}$. The $\beta$-D-glucose amounts to approx. $64 \%$ of the total glucose, depending on the ambient conditions such as $\mathrm{pH}$ and buffer composition. In a volume of $100 \mathrm{ml}$, the glucose concentra- tion can be reduced by up to $1 \%$ of the total due to glucose consumption by the sensor. These aspects have to be considered when handling smaller volumes where the influence might become measurable.

\subsection{Measurements of $\beta-D-$ Glucose, L-Lactate and L-Glutamate in the Bioreactor}

Only the results of glucose measurements in an early phase of commercialization of the sensor are described below. The experiments were completed at the University of Applied Science in Wädenswil (see acknowledgement ${ }^{[12]}$ ) and the results are illustrated in Fig. 3.

After adding the biosensor to the bioreactor mounted on its cover, the sensor is calibrated to the initial glucose concentration in the cell culture medium after a conditioning time of approx. $30 \mathrm{~min}$. The second calibration point is at zero glucose concentration by default.

In one experiment, the glucose sensor was mounted on a T-Flask bioreactor inoculated with a culture of $\mathrm{CHO} \mathrm{K} 1$ cells, and the glucose concentration was monitored directly and continuously with the glucose sensor (red and black lines). However, in order to compare the various methods of glucose analysis, ${ }^{[12 b]}$ samples of each batch were collected daily and analyzed by the instrument 'Bioprofile' [4] (triangles) and by HPLC (squares). In addition, the compatibility of the sensor with the $\mathrm{CHO}$ cells was investigated.[11] The user was able to handle the system after a short introduction, and the integrity of the system was sustained over a two-week period. The deviation between the sensor and the ref- 
erence methods was approx. $0.16 \pm 0.09$ $\mathrm{g} \mathrm{L}^{-1}$ glucose for HPLC and $0.14 \pm 0.13$ $\mathrm{g} \mathrm{L}^{-1}$ for the Bioprofile analyzer. Overall the mean difference between the reference method and the sensor was of the same order of magnitude as that between the reference methods themselves.

In another experiment (results not shown here), a CITSens Bio sensor was mounted on a Superspinner, in which Chinese hamster ovary ( $\mathrm{CHO}$ ) cells were grown in suspension. The infrastructure for the experiments was kindly provided by ZHAW (see acknowledgement).

After the conditioning period of 30-60 min required by the sensor, the decrease in glucose concentration was clearly visible. On the addition of glucose after $48 \mathrm{~h}$ (feed cycle), the glucose concentration instantaneously rose prior to decreasing again as the carbohydrate source is digested.

\section{Results and Specifications}

\subsection{Metrology and Method Comparison}

A glucose-specific biosensor provided by C-CIT AG is used at the Federal Office of Metrology METAS in a chronoamperometric system in order to establish an infrastructure for electroanalytical chemistry. The goal is to provide a valuable contribution to the traceability of glucose content measurements by biosensors. [10] In the clinical chemistry laboratory, various types of instruments detect and report fundamentally different glucose quantities. In the high-throughput laboratory glucose measurements are primarily performed photometrically. Self-monitoring and point-of-care testing devices use direct reading biosensor devices that do not need dilution of the sample. In order to compare the results of analytical methods which measure different quantities of glucose, fixed factors were introduced to convert the results.

In biotechnology, the same situation is currently observed. In Fig. 3, three different analytical methods to quantify substrate concentrations are compared, namely: two analysis procedures run externally (HPLC and Bioprofile) and one procedure which determines the substrates in the undiluted cell culture medium without any sample pre-treatment (CITSens Bio). Even though each method is sensitive to another species of the substrate, the results are comparable when referred to the calibration procedure, with a parallel shift in the concentration results between the methods (systematic deviation). This shift corresponds to the correction factor used in clinical chemistry.

Typically, the biosensor reacts to a specific species of the substrate. For GOD, the substrate is $\beta$-D-(+)-glucose. In the case of
D-(+)-glucose as a substrate, two anomers are known which mutarotate between the $\alpha$ - and the $\beta$-anomer in aqueous solution. The $\beta$-anomer is oxidized by the enzyme GOD and relies on the enzyme specifications (see Section 2.2). For glucose as a substrate, this fraction accounts for only $64 \%$ of D-(+)-glucose anomer. ${ }^{[13,14]}$ However, since the sensor is calibrated to D(+)-glucose (see Section 2.1), the results are referred to mass concentration of this species and are comparable to the substrate concentration evaluated by other methods.

Remarkably, the results yielded by measurements made directly in the cell culture medium tend to be higher than those of external methods. This phenomenon is well known from measuring blood directly. Since the sensor responds to the molality of $\beta$-D-(+)-glucose (amount of $\beta$ $D-(+)$-glucose per unit mass of water), the result is independent of the concentration of fats and proteins which contribute to the total volume. In contrast, if the cultivation medium is sampled by a pipette, these components contribute to the sampled total volume and glucose is also referred to the volume of other components than glucose. Therefore, the glucose mass concentration measured after sample pretreatment and dilution with reagents and $\mathrm{pH}$-buffer is referred to a higher volume than that in the undiluted specimen; the concentrations are lower than those measured directly in the specimen within the bioreactor.

In Fig. 3, the deviation between the analytical methods is relatively small. It would be more pronounced in cultures with high cell density and highly concentrated cultivation media. The biosensor's concentration results correlate inversely with the exponential increase in cell density.

\subsection{Biocompatibility}

Toxic effects between the sensor and the cells were investigated by measurements of the cell density and cell vitality. No toxic effects due to an interaction between the sensor and the cells were observed (Ce-Power GmbH, CH-8820 Wädenswil). However, the advantage of inline monitoring with the biosensor system, as opposed to the off-line measurement methods, only became apparent after the fourth cycle. Both biosensors indicated the end of cell growth when the glucose concentration remained constant (black line), confirming that no further glucose was being consumed and that the stationary phase of the cells had been reached. This stationary phase was not readily identified by the daily off-line measurement using the reference method.

In addition to measuring the rate of cell growth and multiplication, continuous analysis also enables immediate detection of any contamination, which causes the glucose concentration to decrease instantaneously.

\subsection{Bioburden Testing for Medical Devices}

In March 2010, three glucose-specific sensors CITSens Bio were tested for their 'bioburden' according to SN ISO 117371 and European Pharmacopeia ch.2.6.12 by Medistri SA, Domdidier, Switzerland (www.medistri.com). The tests were executed in Trypcase Soja and Thio Clair Bouillon at 24 and $33{ }^{\circ} \mathrm{C}$. No growth induced by the biosensors was detected, the sensors were considered 'conform' according to the standards mentioned above and USP.

\subsection{Specifications for Monitoring $\beta-D-G l u c o s e$}

The sensor can be purchased as an add-on and can be connected to any disposable reactor with a volume of $100 \mathrm{ml}$ or more (see Fig.1a and 1b, Fig. 2). The shelf life of the sensors is 6 months at 4 ${ }^{\circ} \mathrm{C}$. The consumption of $\beta$-D-glucose in a medium is $<10^{-12} \mathrm{~mol}$ per $\mathrm{s}$. The detection limit (6 SD) is $0.18 \mathrm{~g}$ glucose $\mathrm{l}^{-1}$, which is equivalent to $1 \mathrm{mmol} \mathrm{l}^{-1}$. The operating range is $0.18-10.8 \mathrm{~g} \mathrm{l}^{-1}$, equivalent to $1-60 \mathrm{mmol} \mathrm{l}^{-1}$. The guaranteed operating lifetime is 14 days.

Application recommendation: The sensor has to be operated in a biologically buffered medium under stable temperature conditions. Under other conditions its operation cannot be guaranteed.

\subsection{Specifications for Monitoring L-Glutamate and L-Lactate}

The shelf life of the sensors is 6 months at $4{ }^{\circ} \mathrm{C}$. The detection limit (6 SD) is at 1 mmol $\mathrm{l}^{-1}$ equivalent to $0.147 \mathrm{~g} \mathrm{~L}$-glutamate $\mathrm{I}^{-1}$ and $0.09 \mathrm{~g} \mathrm{~L}$-lactate. The operating range is similar to glucose and is between 1 and $60 \mathrm{mmol} \mathrm{l}^{-1}$ which is up to $5.4 \mathrm{~g} \mathrm{l}^{-1} \mathrm{~L}$-lactate and $8.8 \mathrm{~g} \mathrm{l}^{-1} \mathrm{~L}$-glutamate. The guaranteed operating lifetime is 14 days.

\section{Summary}

The use of an in-line biosensor has confirmed the effectiveness of continuous monitoring in controlling the quality of bio-processes, as stipulated by the FDA in their Guidance for Industry to PAT (process analysis).

In-line sensors offer several advantages including:

- elimination of sampling steps

- reduced risk of contamination

- no reagent costs, unlike with batch analyzers

- chemical analyses can be carried out close to the process, with the process control

- centralized 
- immediate detection of contaminations and any other process changes.

- The results of the glucose biosensor can be traced back to a standard run at the Federal Office of Metrology (METAS), Switzerland, for quality assessment.

\section{Conclusions}

The biosensor system described in this article has been developed specifically for bioprocess monitoring and fulfills the requirements of this application. The sensor is able to work continuously for 14 days and provides data to survey glucose consumption related to increasing cell density. The instrumentation and the biosensors are available at a reasonable price and help to make the production process very effective (see $w w w . c$-cit.ch). In addition, continuous monitoring saves resources. The production process can be stopped in the phase when the rate of product yield and cell density are at a maximum. Moreover, continuous monitoring reduces the risk of contamination. Staff do not have to spend time sampling aliquots of the medium in the flow-bench for external analysis, and the production process is not interrupted by having to remove the disposable reactor from the incubator.

\section{Acknowledgements}

The support of Prof. Regine Eible at the University of Applied Sciences, Wädenswil (ZHAW), who allowed the authors to carry out experiments in disposable reactors, is gratefully acknowledged. We are grateful for the supply of L-glutamate oxidase by Prof. Kusakabe at Tsukuba University, Japan.

Received: September 6, 2010
[1] U.S. Department of Health and Human Services et al., Pharmaceutical CGMPs, September 2004, Guidance for Industry, PAT - A Framework for Innovative Pharmaceutical Development, Manufacturing, and Quality Assurance, September 2004.

[2] A. Heller, B. Feldmann, Chem. Rev. 2008, 108, 2482.

[3] YSI STAT 2300, YSI 2700 SELECT et al., YSI Life Sciences, www.ysilifesciences.com.

[4] BioProfile ${ }^{\circledR}$, Automated Chemistry Analyzer, Nova Biomedical Corporation, www.novabiomedical.com.

[5] A. Pellisier, U. E. Spichiger-Keller, Project Report Center for Chemical Sensors, ETH Zürich-Technopark, July 2007.

[6] a) F. Messi, Ph.D. thesis, Swiss Federal Inst. of Technology (ETH), Zürich, Nr. 9559, 1991; b) Cited in: Zang et al., Bio/Technology 1995, 13, 389.

[7] Enzyme-Sensor Corporation, 311, Tsukuba Industrial Liaison and Cooperative Research Center, Tsukuba University, 1-1-1 Tennodai, Tsukuba, Ibaraki 305-8577, Japan.

[8] J. Arima, C. Sasaki, C. Sakaguchi, H. Mizuno, T. Tamura, A. Kashima, H. Kusakabe, S. Sugio, K. Inagaki, FEBS J. 2009, 1-10, submitted.

[9] Albis Technologies AG, CH-8047 Zürich.

[10] S. Wunderli, H.-P. Andres, Electroanalysis 2009, 21, 1984

[11] T. Schopper, Diploma work, Zürcher Fachhochschule Wädenswil (ZHAW), 2007, confidential.

[12] a) D. Eibl, T. Peuker, R. Eibl, in 'Single Use Technology in Pharmaceutical Manufacture', Eds. D. Eibl, R. Eible, John Wiley \& Sons, Inc., 2009; b) S. Spichiger, U. E. Spichiger-Keller, in 'Single Use Technology in Pharmaceutical Manufacture', Eds. D. Eibl, R. Eible, John Wiley \& Sons, Inc., 2009.

[13] 'Chapter D 2.19 Kohlenhydrate', in 'Lehrbuch der Pharmazeutischen Chemie', Eds H. Auterhoff, J. Knabe, H.-D. Höltje, Wissenschaftliche Verlagsgesellschaft $\mathrm{mbH}$, Stuttgart, 1991, p. 320.

[14] H. S.Lee, J. Hong, J. Biotech. 2000, 84, 145. 